\title{
A Novel Marker Based Method to Teeth Alignment in MRI
}

\author{
Jean-Marc Luukinen ${ }^{1,2}$, Daniel Aalto ${ }^{3,4}$, Jarmo Malinen ${ }^{5}$, Naoko Niikuni ${ }^{1,6}$, Jani Saunavaara ${ }^{7}$, \\ Päivi Jääsaari ${ }^{1,2}$, Antti Ojalammi ${ }^{5}$, Riitta Parkkola ${ }^{7}$, Tero Soukka ${ }^{1,2}$, and Risto-Pekka Happonen ${ }^{1,2}$ \\ ${ }^{1}$ Department of Oral and Maxillofacial Surgery, University of Turku, Lemminkäisenkatu 2, 20520, Turku, Finland \\ ${ }^{2}$ Department of Oral and Maxillofacial Diseases, Turku University Hospital, Lemminkäisenkatu 2, 20520, Turku, Finland \\ ${ }^{3}$ Institute for Reconstructive Sciences in Medicine, Misericordia Hospital, 1694087 Ave NW, Edmonton, AB, Canada \\ ${ }^{4}$ Department of Communication Sciences and Disorders, University of Alberta, 8205114 St NW, Edmonton, Canada \\ ${ }^{5}$ Department of Mathematics and System Analysis, Aalto University, Otakaari 1 F, 00076, Espoo, Finland \\ ${ }^{6}$ Department of Pediatric Dentistry, Nihon University, School of Dentistry, 4-8-24 Kudan-Minami, Chiyoda-ku, Tokyo, \\ Japan \\ ${ }^{7}$ Department of Medical Physics, Turku University Hospital, Kiinamyllynkatu 4-8, 20521, Turku, Finland \\ Corresponding author Jean-Marc Luukinen: jean-marc.luukinen@utu.fi
}

\begin{abstract}
Magnetic resonance imaging (MRI) can precisely capture the anatomy of the vocal tract. However, the crowns of teeth are not visible in standard MRI scans. In this study, a marker-based teeth alignment method is presented and evaluated. Ten patients undergoing orthognathic surgery were enrolled. Supraglottal airways were imaged preoperatively using structural MRI. MRI visible markers were developed, and they were attached to maxillary teeth and corresponding locations on the dental casts. Repeated measurements of intermarker distances in MRI and in a replica model was compared using linear regression analysis. Dental cast MRI and corresponding caliper measurements did not differ significantly. In contrast, the marker locations in vivo differed somewhat from the dental cast measurements likely due to marker placement inaccuracies. The markers were clearly visible in MRI and allowed for dental models to be aligned to head and neck MRI scans.
\end{abstract}

Keywords: Dental imaging, Magnetic resonance imaging, MRI markers, teeth superposition, vocal tract imaging.

\section{INTRODUCTION}

Computed tomography (CT) is the clinical standard for creating 3D digital models of jaws. [1], [2] However, X-ray based methods expose the patients to relatively high radiation doses, which restricts their use for studying speech production. [3]-[6] Magnetic resonance imaging (MRI) provides an attractive alternative to CT since it can provide both static and dynamic imaging without the associated high ionizing radiation dose of the latter. [7]-[9] While the soft tissues are well captured in standard MRI, the high mineral content teeth crowns are typically not visible. Complete MRI based models of oral anatomy benefit the study of not only speech but also the study of other functions like chewing. In this publication, a marker-based teeth alignment method is presented and evaluated.

Methods to visualize teeth and their relation to the skull in MRI can be roughly classified in two groups. First, a digital model of dentition can be aligned to the MRI by completely or partly covering the dentition with a contrast medium to provide data for alignment. [10], [12]-[18] In these methods, a digital dental model based on optical or conventional impressions is superimposed to the MR image. Second, the dentition can be directly measured by MRI. [11], [19], [20]
The methods that have been used earlier will be presented briefly in the following paragraph.

Pure water [15] and blueberry juice [12], [16], [20] have been used as a contrast medium to cover the dentition completely. Clinical applicability is limited by an elevated aspiration risk which can be minimized by using paired plate containers [10], thermoplastic elastomer mouthpiece [13], retainer filled with ferric ammonium citrate jelly [14], or molded silicone mouthpiece coated with petroleum jelly [17]. However, hypersensitivity to a contrast medium may be a restricting factor. [21] Even a body part like the tongue can act as a contrast medium. [18] Nevertheless, tight lingual frenulum, reduced tongue mobility or small tongue size, e.g., due to glossectomy, can restrict the applicability of the method. Extraorally placed MRI visible markers have been proposed to solve the alignment problem. However, when the markers are attached to the facial skin, they are sensitive to the movement of the mimetic muscles. [17] Finally, customized MRI sequences have been proposed to simultaneously image hard and soft tissues, but those specialized sequences are not available on standard clinical scanners. [11], [19], [20], [22] 


\section{SUBJECTS \& METHODS}

\section{Patients}

Ten patients ( 6 men and 4 women) undergoing orthognathic surgery treatment were recruited. According to the clinical standards of the hospital, the surgical operations were planned based on cephalograms, and CT scans were not clinically justified. The youngest patient was 19 and the oldest 47 years old (Table 1.). Each patient signed an informed consent form. The procedure was approved by the Ethical Board of the Hospital District of Southwest Finland.

Table 1. Descriptive statistics of the patients participating in the study. Mean distance measures ( \pm standard deviation) based on structural MRI: I-PNS (incisor-posterior nasal spine), M-S (molarsella), and W (width dd 16-26).

\begin{tabular}{llll}
\hline & Men & Women & All \\
\hline Participants & 6 & 4 & 10 \\
Age (years) & $34 \pm 9$ & $27 \pm 10$ & $31 \pm 9$ \\
I-PNS (mm) & $61.0 \pm 6.9$ & $54.3 \pm 4.8$ & $58.8 \pm 6.9$ \\
M-S (mm) & $82.4 \pm 6.7$ & $75.3 \pm 4.5$ & $79.3 \pm 6.4$ \\
W (mm) & $54.9 \pm 3.2$ & $50.5 \pm 2.7$ & $52.9 \pm 3.7$ \\
\hline
\end{tabular}

\section{Dental casts}

Dental casts and a bite index were taken for each patient. [23] Central occlusion was recorded using a bite index (made of beeswax). The bite index was also used in MRI to ensure central occlusion.

\section{Markers}

Custom-made MRI-visible markers (Fig.1.a) were attached to the teeth on the buccal/labial side of first maxillary incisors (dd 11 and 21) and molars (dd 16 and 26). The markers were also attached to the corresponding places on the dental casts (Fig.1.b). An orthodontic instrument (a Boone bracket positioning gauge) was used for vertical and caliper for horizontal placement. The exact place varied between the patients to make sure that the markers did not disturb the occlusion. An adhesive (Scotchbond TM Universal) was chosen to attach the markers rigidly to the dental surface with minimal risk of damage to the enamel surface during removal. Markers were attached to dental casts with a two-component adhesive (Araldite Rapid, Huntsman).

The MRI markers that were used had a flattened half spherical shape of diameter $5.0 \pm 0.3 \mathrm{~mm}$ and thickness of 3.0 $\pm 0.3 \mathrm{~mm}$. The markers had a flat facet to facilitate attachment to a dental surface using UV hardening dental adhesive.

The MRI signal from the marker is due to vegetable oil (food quality canola oil) whose most abundant fatty acid components are in the fluid phase at room temperature. The oil is bound to a matrix, produced from a circular aspen wood (Populus tremula L.) disk of diameter $3.8 \pm 0.1 \mathrm{~mm}$ and thickness $2.0 \pm 0.0 \mathrm{~mm}$.

To produce the disks, furniture quality aspen blocks were first slowly air dried in a slightly elevated room temperature as is usual in all woodworking. When the material was deemed dry enough for cutting, wooden circular disks were produced in batches of several hundreds of items. Another heat treatment is required to drive out the remaining moisture, and it was carried out by heating the disks using an infrared lamp for about 15 minutes. Since the temperature measurement of such small objects is difficult, the desiccation process was only visually observed so that the wood material would not get carbonized, thereby destroying the capillary structure. The weight of the disks was measured several times during the process, and the disks were deemed ready when no decrease in weight could be observed by additional infrared irradiation. It was observed that the weight of a successfully desiccated disk would soon rise markedly if it was left in the analytical balance unprotected against the humidity of air. After having removed practically all water from the wood material, vegetable oil is introduced without delay into its capillary structure by immersion. Immersion time of several hours was used. Finally, between 51-55 \% of the marker matrix weight (with reachable maximum at $57 \%$ of weight) consists of MRI visible oil.

The coating of the markers is obtained by dipping the markers in Bisphenol A -based epoxy resin. Before applying the coating, the marker matrices were rounded in a sanding drum to remove sharp corners where the coating would peel. The viscosity and surface tension of the epoxy resin were adjusted by adding water-free ethanol. It was observed that even a small amount of water in the mixture would make the resin grainy and unusable. In our application, the thickness of the coating is between $0.1-1.0 \mathrm{~mm}$ depending on the surface of the marker in question. The non-uniform thickness is due to the surface tension of the unhardened coating and the fact that the marker matrix was lying on a flat surface during hardening. The thin part of the coating is on the flat surface of the marker; it is positioned against the tooth surface where the thickness of the coating plays a significant role.

Because of the layered structure, the markers have good MRI contrast to air and osseous structures (due to their vegetable oil content) as well as to the water or fat containing tissues (due to their epoxy coating) (Fig.1.c).

The MRI relaxation time parameters of vegetable oil, or the prepared MRI visible marker matrices, were not measured. It was, however, observed in MRI phantom experiments that the coated markers produce a signal level comparable with oil containing gelatin capsules that are often used to mark positions on patients' skin during MRI experiments.

\section{Patient and dental cast imaging}

All MRI scans were performed using Siemens Magnetom Avanto 1.5T scanner (Siemens Healthineers, Erlangen, Germany). A 12-element Head Matrix Coil and a 4-element Neck Matrix Coil were used to cover the anatomy of interest. The coil configuration allows the use of Generalized Autocalibrating Partially Parallel Acquisition (GRAPPA) technique to accelerate acquisition. 3D VIBE (Volumetric Interpolated Breath-hold Examination) MRI sequence was used to acquire the high-quality images of the patients' maxilla and mandible. Following parameters were used to obtain images with $0.9 \mathrm{~mm}$ isotropic voxels in 215 seconds: Time of repetition (TR) was $4.26 \mathrm{~ms}$, echo time (TE) $1.57 \mathrm{~ms}$, flip angle (FA) $6^{\circ}$, parallel acquisition technique (PAT) acceleration factor, number of slices 120 , and number of averages 3 . 
For comparison, a data set with $1.8 \mathrm{~mm}$ isotropic voxels was acquired during sustained vowel production. Using VIBE sequence and the following parameters the data acquisition took less than 9 seconds: TR $3.75 \mathrm{~ms}$, TE $1.19 \mathrm{~ms}$, FA $6^{\circ}$, PAT 2, number of slices 44 , and number of averages 2 .

In addition, 3D MRI technique was used to scan the dental casts with the markers. Since dental casts are not visible in MRI, they were embedded in blueberry juice that served as a contrast medium for MRI (Fig.1.d). The following parameters allowed imaging with $0.9 \mathrm{~mm}$ isotropic voxels in less than $3 \mathrm{~min}$ : TR $4.26 \mathrm{~ms}$, TE $1.57 \mathrm{~ms}$, FA $6^{\circ}$, number of slices 120 , and number of averages 4 .
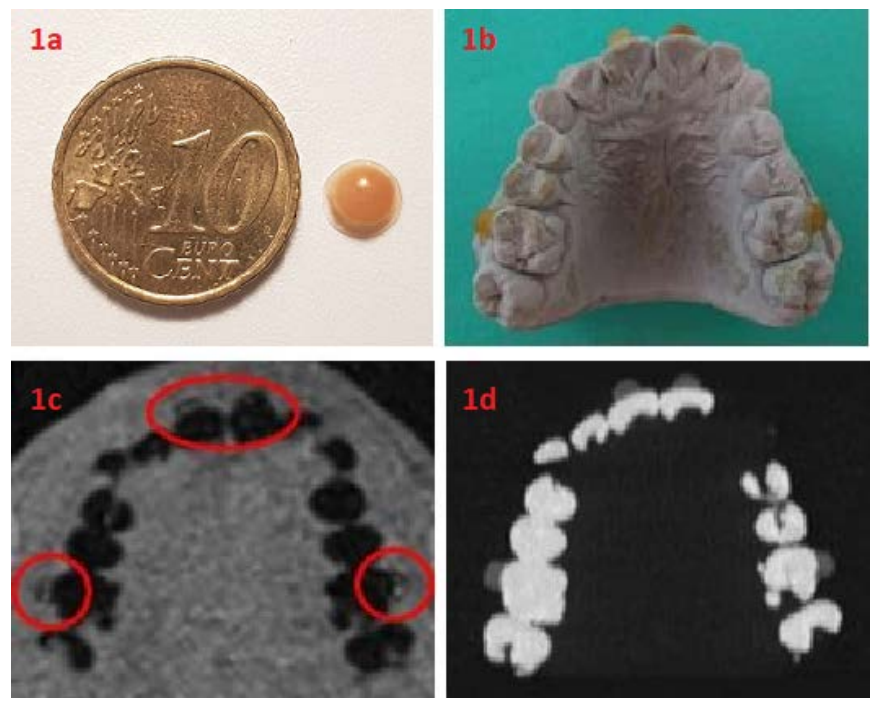

Fig.1. a) A marker with a ten cent (euro) coin with diameter $19 \mathrm{~mm}$, b) An inferior view of the dental cast showing the markers on dental cast, c) The corresponding axial slice of the patient showing markers, d) Axial slice of an MR image showing dental cast immersed in blueberry juice.

Cephalometry (Cranex ${ }^{\circledR}$ D, Soredex, Tuusula, Finland) radiographs were used as a benchmark for the teeth alignment method evaluation. The imaging time in cephalogram was 14.6 s. During the imaging, the head was stabilized to avoid movement artifact.

\section{Landmarks}

Three cranial landmarks visible in both imaging modalities (X-ray and MR) were selected. These were anterior nasal spine (ANS), posterior nasal spine (PNS), and the midpoint of sella (S). Dental markers in the MRI and the corresponding points on the teeth in lateral X-ray served as additional landmarks.

\section{Landmark and marker registration}

Intermarker distances were obtained from the patient MRI, dental cast MRI, and from dental casts using a caliper. The anatomical landmarks were measured from the cephalogram and the patient MRI. The measurements between different modalities were compared.
The landmarks were located in the MR image by adjusting the orientation and position of the mutually orthogonal planes in the orthogonal multi-planar reconstruction (MPR) and recording the coordinate values using OsiriX (Advanced Open-Source PACS Workstation DICOM Viewer) (Fig.2.).
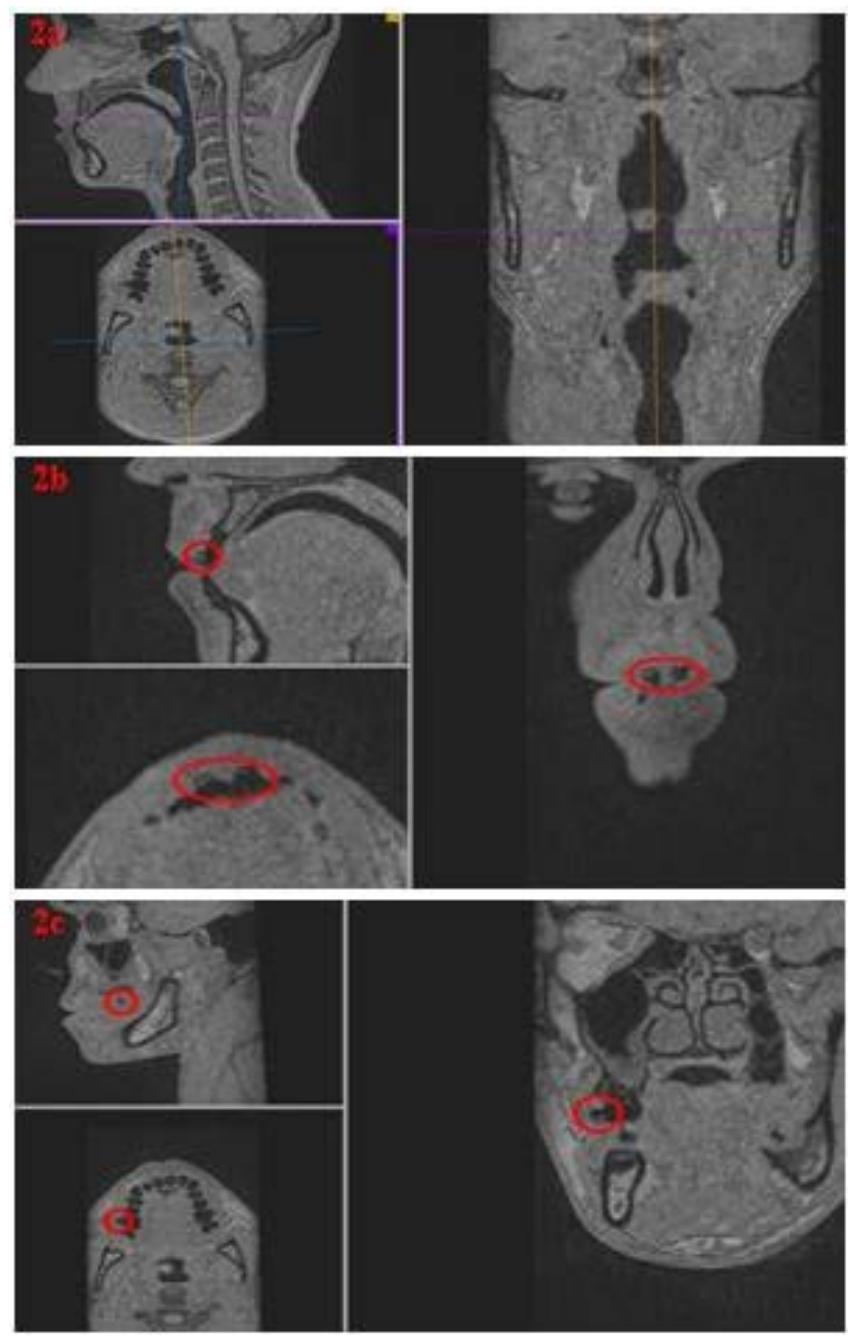

Fig.2. a) The MPR technique was used to locate anatomical landmarks in the midsagittal plane, b) Markers on the maxillary incisors (dd 11, 21), c) The marker on the first maxillary molar (d 16).

The midsagittal plane was aligned based on the foramen magnum or four uppermost vertebrae C1-C4, the eyes, and the palatal arch. The dental markers were registered at planes tangent to the flat surface of the markers. Within the dental casts, the distances between the dental markers as well as the vertical position between the markers and the inferior edge of the crown were measured by a digital vernier caliper (Mitsutoyo, nominal accuracy $0.1 \mathrm{~mm}$ ). Measured distances between the teeth were dd 11-21, dd 11-16, dd 11-26, dd 2126, dd 21-16, and dd 16-26 (Fig.3.). Three independent measurements were taken for each measure, and the median value was used in geometrical transformations and statistical analyses. For estimating measurement errors, measurements for one patient were repeated 20 times. 


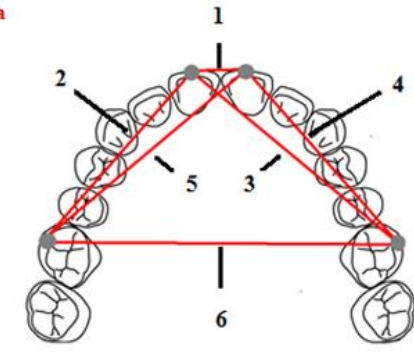

3b

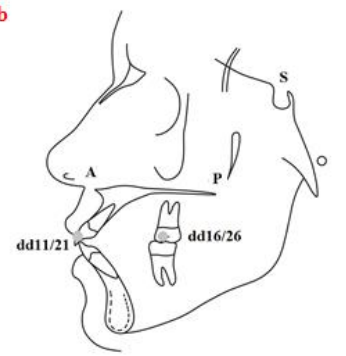

$5 a$
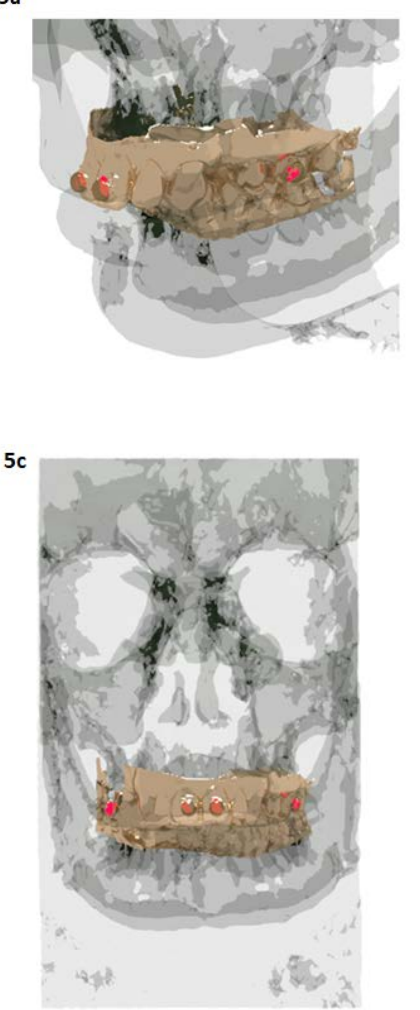
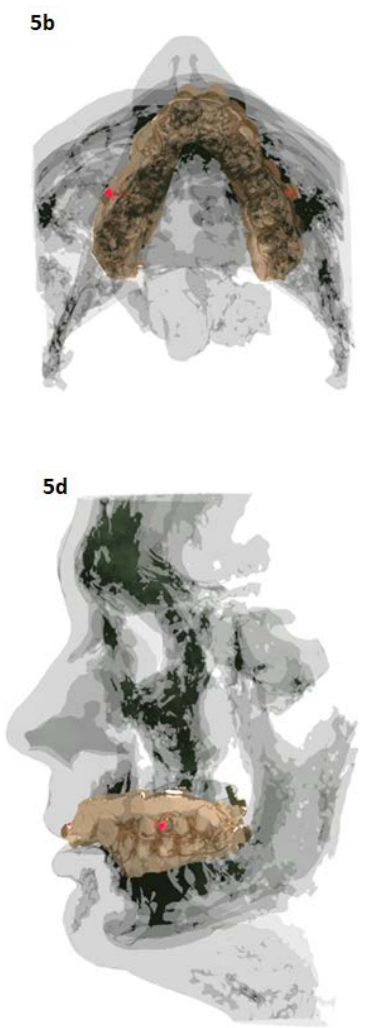

Fig.3. a) Schematic drawing of the distances between the dental markers: 1 = dd 11-21, 2 = dd 11-16, $3=$ dd 11-26, 4 = dd 21-26, $5=$ dd 21-16, and $6=$ dd 16-26, b) Illustration of the anatomical landmarks and the markers: $\mathrm{A}=$ Anterior nasal spine, $\mathrm{P}=$ Posterior nasal spine, and $\mathrm{S}=$ Sella.

During the X-ray imaging, the markers were not held in the mouth. By observing marker positions on the dental casts, the corresponding points were registered in the cephalogram. To compare the cephalogram and the MRI data, the MRI data was geometrically transformed to match the corresponding cephalometric distances (Fig.4.). The transformation was very coarse: parasagittal points (incisors, molars) were orthogonally projected to the midsagittal plane (defined as the plane containing ANS, PNS, and sella).
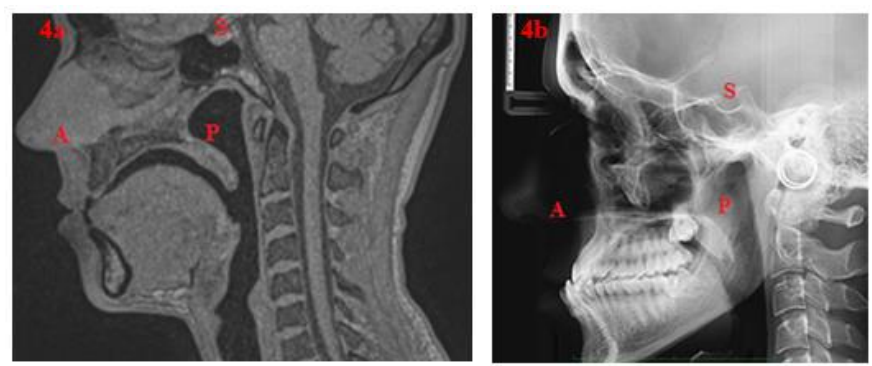

Fig.4. a) A midsagittal slice of an MRI, b) Cephalogram. In both pictures, from the same patient the anatomical landmarks ( $\mathrm{A}=$ Anterior nasal spine, $\mathrm{P}=$ Posterior nasal spine, and $\mathrm{S}=$ Sella) are clearly observable.

\section{Dental cast alignment}

The recorded coordinate values of the markers on the dental cast in MRI and the corresponding coordinate values on the dentitions in MRI were used to create an optimal estimate of the rigid body coordinate transformation based on the singular value decomposition (SVD). [24] The alignment results are presented visually in Fig.5. and summarized in Table 2.

Patients M04 and M06 show subvoxel accuracy, Table 2. Some markers became loose (d26 in M07 and d16 in W05) or were lost completely (d16 in M07). At least three markers are necessary for alignment and due to missing markers could not be done for M03 and W04. *For M02 the markers were attached to $\mathrm{d} 13$ and $\mathrm{d} 23$ instead of $\mathrm{d} 11$ and $\mathrm{d} 21$. **The marker d16 became loose (and consequently misplaced) and was not included in the computation of the optimal rigid transformation.
Fig.5. Translucent 3D models of the head and neck anatomy with dark color for osseous tissue. The dental casts (light brown) are superposed to the images based on the markers (red). The oblique view shows an overbite a), the inferior view of the maxilla shows the lateral asymmetry b), frontal view shows lateral asymmetry in mandible c), and the lateral view d) shows the position of the marker on the molar.

Table 2. Alignment error between optimally rotated and translated dental cast model markers and the intraoral markers.

\begin{tabular}{|l|l|l|l|l|}
\hline \multicolumn{5}{|c|}{ Alignment error (mm) } \\
\hline Patient & $\mathrm{d} 11$ & $\mathrm{~d} 16$ & $\mathrm{~d} 21$ & $\mathrm{~d} 26$ \\
\hline M02* & 1.61 & 1.62 & 0.88 & 1.28 \\
\hline M03 & N/A & N/A & N/A & N/A \\
\hline M04 & 0.40 & 0.28 & 0.60 & 0.69 \\
\hline M06 & 0.43 & 0.64 & 0.62 & 0.75 \\
\hline M07 & 3.53 & N/A & 1.91 & 3.68 \\
\hline M08 & 2.41 & 1.91 & 2.57 & 0.89 \\
\hline W04 & N/A & N/A & N/A & N/A \\
\hline W05 & 0.12 & $7.52 * *$ & 0.24 & 0.12 \\
\hline W06 & 0.26 & 0.77 & 1.11 & 0.65 \\
\hline W07 & 1.02 & 0.87 & 0.95 & 0.82 \\
\hline
\end{tabular}




\section{Statistical analysis}

The data was analyzed for consistency within every modality and for discrepancy across modalities based on distance measures. Caliper precision was defined as the residual standard error of the linear regression model with intermarker distance as the dependent variable and the marker pair as the independent variable. MRI registration precision for dental markers (including both dental cast and patient MRI) and cranial landmarks was defined similarly. Cephalometric registration precision was estimated similarly.

Cranial landmarks offer an alternative to marker-based solutions. The accuracy of locating the landmarks was assessed using three cranial landmarks (ANS, PNS and sella) using repeatedly acquired MRI scans with two spatial resolutions $(0.9 \mathrm{~mm}$ isotropic voxels, $1.8 \mathrm{~mm}$ isotropic voxels). [25], [26] The consistency was again defined as the standard residual error of the fitted linear regression model.

Cross-modally, caliper measurements were compared with the MRI measurements of dental casts and patient MRI. A linear regression model for the dental marker distance differences was fitted, and the standard residual error and the intercept served as a measure for precision and bias. More detailed models included the distance measures as a factor. Similarly, linear regression models were used to compare cephalometric and MRI measures between cranial landmarks. Finally, dental alignment was assessed by linear regression models where the dependent variable was the difference between MRI and cephalometric landmark to marker distance.

\section{Visualization of results}

The results were visualized in one patient. Accurate coordinate measurements were made from the dental markers in MRI from the dental cast immersed in blueberry juice and the patient. Based on these coordinates, geometric models were created using the marching cubes algorithm (Fig.5.). [27], [28].

\section{RESULTS}

\section{Variability within a modality}

Caliper precision was $0.15 \mathrm{~mm}$. MRI registration precision for dental markers attached to dental cast was $0.32 \mathrm{~mm}$ and $0.39 \mathrm{~mm}$ when attached to the dentition. The MRI registration precision for the cranial landmarks was $0.57 \mathrm{~mm}$ and the cephalometric landmark registration precision was $0.32 \mathrm{~mm}$ (Fig.6.). In repeated MRI scans (1.8 mm isotropic voxels) of the same subjects, the cranial landmark registration precision was $1.2 \mathrm{~mm}$.

\section{Cross modal comparisons}

Dental cast MRI did not differ significantly from the corresponding caliper measurements (intercept: $0.47 \mathrm{~mm}$, residual standard error: $1.8 \mathrm{~mm}, \mathrm{t}=1.8$, n.s.). The dental MRI distances were underestimated with respect to the caliper measurements (intercept: $0.77 \mathrm{~mm}$, residual standard error: $1.3, \mathrm{t}=4.1, \mathrm{p}<0.01)$. The underestimation was mainly due to dd 21-26 distance $(1.6 \mathrm{~mm}, \mathrm{t}=2.8, \mathrm{p}<0.01)$ and dd $11-$
$16(1.5 \mathrm{~mm}, \mathrm{t}=2.7, \mathrm{p}=0.01)$. Differences in intermarker distances between dental cast MRI and patient dental MRI measurements were not statistically significant (intercept: $0.0 \mathrm{~mm}$, residual error: $2.0 \mathrm{~mm}, \mathrm{t}=0.1$, n.s.). Cranial landmark accuracy was good (intercept $0.6 \mathrm{~mm}$, residual standard error: $3.0 \mathrm{~mm}, \mathrm{t}=1.0$, n.s.). The distance between dental markers and cranial landmarks was in average $2.1 \pm$ $2.9 \mathrm{~mm}$ larger in MRI compared to cephalogram $(\mathrm{t}=4.9$, $\mathrm{df}$ $=47, \mathrm{p}<0.01)$. The molar-PNS distance was $3.0 \mathrm{~mm}$ larger $(\mathrm{p}=0.07)$ and the molar-ANS distance $3.6 \mathrm{~mm}$ smaller $(\mathrm{p}=$ 0.01 ) compared with the average $2.1 \mathrm{~mm}$ general overestimation trend.

\section{Dental cast alignment}

The alignment was successful in eight cases and impossible in two cases due to missing data. The difference between the optimally aligned cast and the dental markers varied around one millimeter as shown in Table 2. For M03 and W04, at least two markers were missing preventing alignment. The molar marker (d16) became loose in M07 when entering MRI and could not be reattached in situ while the other molar marker (d26) became loose and displaced within MRI. The resulting rigid transformation contained a reflection and led to erroneous alignment. In W05, the marker d16 became loose and displaced and was not included when determining the rigid transformation. Seven alignments were successful, an example is shown in Fig.5.

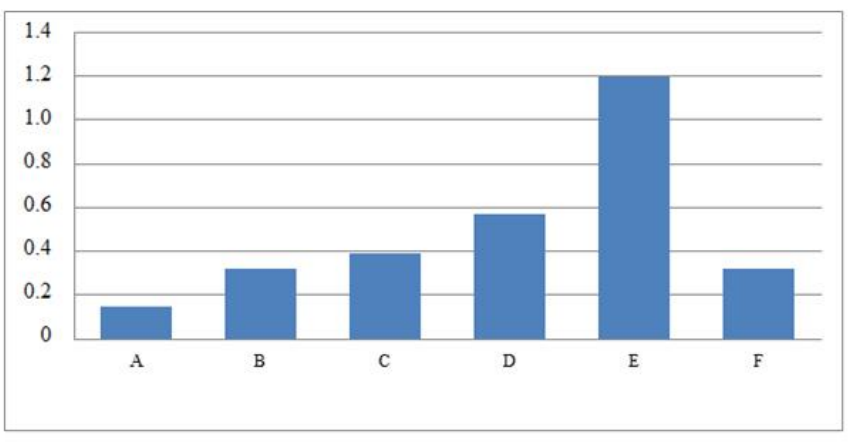

Fig.6. Standard deviations (in $\mathrm{mm}$ ) of repeated measurements for each measuring method.

A. Caliper measurement from the dental cast.

B. Dental cast in MRI with $0.9 \mathrm{~mm}$ isotropic voxels.

C. Dentition in MRI with $0.9 \mathrm{~mm}$ isotropic voxels.

D. Cranial landmarks in MRI with $0.9 \mathrm{~mm}$ isotropic voxels.

E. Cranial landmarks in MRI with $1.8 \mathrm{~mm}$ isotropic voxels.

F. Cranial landmarks in cephalogram.

\section{DISCUSSION}

In this publication, a marker-based method to superpose teeth in MRI is presented and evaluated. Several methods to visualize teeth in MRI have been proposed but the teeth alignment methods have been evaluated only perceptually. In this study, the accuracy is determined by repeated measurements and cross modal comparisons. Based on standard deviation of repeated measurements the accuracy for dental markers in MRI, when attached to dental cast and dentition, is $0.32 \mathrm{~mm}$ and $0.39 \mathrm{~mm}$, respectively. 
Dental MRI marker distances are smaller than the corresponding caliper measurements in the dental casts. The difference is due to ipsilateral comparisons. The poor visibility and moist conditions, especially in molar area, may complicate the attaching of the marker and this way affects the results negatively. This is supported by dental cast caliper and dental cast MRI distances being comparable. On the other hand, the bottom of the marker in MRI may be detected slightly too mesially.

Cranial landmark distances in MRI are comparable to the corresponding cephalometric measurements. The distortion is minor between different imaging methods because the anatomical landmarks were in the midsagittal plane although some of the patients had lateral asymmetries so that the midsagittal plane could not be determined unambiguously.

Even in the same MR stack the detectability of anatomical landmarks is weaker than the detectability of the markers. Firstly, the determination of the midsagittal plane is more difficult than the determination of the marker attachment plane. Secondly, the anatomical shapes (ANS, PNS, and sella) are less symmetrical than the proposed markers which complicates the within plane positioning of the target points.

The measures between markers in 3D MRI and 2D cephalogram showed clear discrepancies. Cephalometric distortion occurs because of different magnification between adjacent parasagittal planes. In MRI, the distances between the anatomical landmarks and markers are systematically $2 \mathrm{~mm}$ bigger than in cephalogram. In a cephalogram, the incisors and molars are not situated in the midsagittal plane and thus give a dual image on the radiograph. [26] The error is due to the fact that in cephalograms the supposed locations of markers are estimated too cranially due to the double images. The cephalometric distances are simulated from the MRI by orthogonally projecting the parasagittal marker locations to the mid-sagittal plane which is not an accurate enough model of the cephalogram. However, the exact orientation of the patient with respect to the image plane and the signal source could not be reliably estimated.

The markers were coated by inert plastic for several reasons. Firstly, the oil will little by little seep out of the matrix if there is nothing to stop it. Secondly, the dental resin does not stick to an oily surface. Thirdly, even though the oil impregnated matrix has good MRI contrast against osseous tissue and air, it has poor contrast against soft tissues such as lip and tongue that may touch the tooth and marker surface.

Handling of the hemispherical markers is cumbersome and the marker adherence is not optimal. A custom applicator could make the maneuvering steadier and increase the precision. The markers are attached to the teeth firmly, but some of the markers would come loose if the patient touched them with the tongue or made big lip movements. Nevertheless, even if a marker came loose, the registration is still possible with the remaining three markers. Furthermore, since the proposed markers are attached to the buccal surface of the teeth, there is no distortion in occlusion. However, some types of malocclusion may complicate or even make the attachment of the marker to the labial/buccal surface of the teeth impossible.

Several unsuccessful ideas were tested in the marker design. Small plastic disks were chemically silver-plated in hopes of getting identifiable MRI artefacts such as those produced by amalgam fillings. Objects containing solid hydrocarbons (e.g., paraffin wax and stearin) were tried but no signal was obtained. Liquid oil can be enclosed in small PLA/ABS plastic frames but producing sufficiently small, dimensionally accurate, and oil tight plastic parts appeared to be impossible, at least using inexpensive 3D printers. Using plastic enclosures of some other type, however, is likely to be a cost-effective solution of choice if large scale production of markers is required. Another idea is to plastic coat gelatin capsules containing oil but the authors considered that approach more uncertain and not much easier to implement than binding the oil to a capillary structure.

The dental markers could be placed and located well for every studied patient despite substantial variability in their occlusions. The accuracy achieved is sufficient to separate the hard and soft tissue. At the moment, these markers are not commercially available, but they could be industrially produced. Even if the proposed markers would not be the solution to the tooth visibility in MRI, they may be needed for the evaluation of another method. The marker data is useful to register the anatomy data which comes from different imaging modalities when complete geometric models are needed.

\section{CONCLUSION}

A marker-based solution to superpose teeth to MRI is presented. The markers are visible in MRI and help in aligning the dental models to head and neck anatomy. The accuracy achieved is promising and suggests that digital dental cast models can be superposed to digital models based on MRI with high precision.

\section{ACKNOWLEDGMENT}

The authors would like to thank anonymous reviewers for valuable feedback, Mr. Jarkko Malinen for significant and time-consuming marker manufacturing, and the funding from Aalto Starting Grant, Magnus Ehrnrooth Foundation, and from Hospital district of Southwest Finland, EVO project grant 13939.

\section{REFERENCES}

[1] Rohner, D., Jaquiéry, C., Kunz, C., Bucher, P., Maas, H., Hammer, B. (2003). Maxillofacial reconstruction with prefabricated osseous free flaps: A 3-year experience with 24 patients. Plastic and Reconstructive Surgery, 112 (3), 748-757.

[2] Plooij, J.M., Maal, T.J., Haers, P., Borstlap, W.A., Kuijpers-Jagtman, A.M., Bergé, S.J. (2011). Digital three-dimensional image fusion processes for planning and evaluating orthodontics and orthognathic surgery. A systematic review. International Journal of Oral and Maxillofacial Surgery, 40 (4), 341-352.

[3] Jones, S. (1929). Radiography and pronunciation. British Journal of Radiology, 2 (15), 149-56.

[4] Scheier, M. (1897). Die Anwendung der Röntgenstrahlen für die Physiologie der Stimme und Sprache. Deutsche Medizinische Wochenschrift, 23 (25), 403. 
[5] Vampola, T., Horáček, J., Laukkanen, A.M., Švec, J.G. (2015). Human vocal tract resonances and the corresponding mode shapes investigated by threedimensional finite-element modelling based on CT measurement. Logopedics Phoniatrics Vocology, 40 (1), 14-23.

[6] Sovijärvi, A. (1938). Die gehaltenen, geflüsterten und gesungenen vokale und nasale der Finnischen sprache - physiologisch-physikalische lautanalysen. Helsinki: Annales Academie Scientiarum Fennicae; German text.

[7] Lingala, S.G., Sutton, B.P., Miquel, M.E., Nayak, K.S. (2016). Recommendations for real-time speech MRI. Journal of Magnetic Resonance Imaging, 43 (1), 28-44.

[8] Baer, T., Gore, J.C., Boyce, S., Nye, P.W. (1987). Application of MRI to the analysis of speech production. Magnetic Resonance Imaging, 5 (1), 1-7.

[9] Scott, A.D., Wylezinska, M., Birch, M.J., Miquel, M.E. (2014). Speech MRI: Morphology and function. Physica Medica: European Journal of Medical Physics, 30 (6), 604-618.

[10] Wakumoto, M., Masaki, S., Dang, J., Honda, K., Shimada, Y., Fujimoto, I., Nakamura, Y. (1997). Visualization of dental crown shape in an MRI-based speech production study. International Journal of Oral and Maxillofacial Surgery, 26, 189-190.

[11] Hövener, J.B., Zwick, S., Leupold, J., Eisenbeiß, A.K., Scheifele, C., Schellenberger, F., Hennig, J., Elverfeldt, D., Ludwig, U. (2012). Dental MRI: Imaging of soft and solid components without ionizing radiation. Journal of Magnetic Resonance Imaging, 36 (4), 841-846.

[12] Hiraishi, K., Narabayashi, I., Fujita, O., Yamamoto, K., Sagami, A., Hisada, Y., Saika, Y., Adachi, I., Hasegawa, H. (1995). Blueberry juice: Preliminary evaluation as an oral contrast agent in gastrointestinal MR imaging. Radiology, 194 (1), 119-123.

[13] Kitamura, T., Nishimoto, H., Fujimoto, I., Shimada, Y. (2011). Dental imaging using a magnetic resonance visible mouthpiece for measurement of vocal tract shape and dimensions. Acoustical Science and Technology, 32 (5), 224-227.

[14] Ng, I.W., Ono, T., Inoue-Arai, M.S., Honda, E., Kurabayashi, T., Moriyama, K. (2011). Application of MRI movie for observation of articulatory movement during a fricative/s/and a plosive/t/ Tooth visualization in MRI. The Angle Orthodontist, 81 (2), 237-244.

[15] Olt, S., Jakob, P.M. (2004). Contrast-enhanced dental MRI for visualization of the teeth and jaw. Magnetic Resonance in Medicine, 52 (1), 174-176.

[16] Takemoto, H., Kitamura, T., Nishimoto, H., Honda, K. (2004). A method of tooth superimposition on MRI data for accurate measurement of vocal tract shape and dimensions. Acoustical Science and Technology, 25 (6), 468-474.

[17] Ventura, S.R., Freitas, D.R., Ramos, I.M., Tavares, J.M.R. (2014). Three-dimensional visualization of teeth by magnetic resonance imaging during speech. In Biodental Engineering II. Taylor \& Francis Group, 1317.
[18] Ventura, S.R., Freitas, D.R., Tavares, J.M.R. (2009). Application of MRI and biomedical engineering in speech production study. Computer Methods in Biomechanics and Biomedical Engineering, 12 (6), 671-681.

[19] Idiyatullin, D., Corum, C., Moeller, S., Prasad, H.S., Garwood, M., Nixdorf, D.R. (2011). Dental magnetic resonance imaging: Making the invisible visible. Journal of Endodontics, 37 (6), 745-752.

[20] Traser, L., Flügge, T.V., Burdumy, M., Kamberger, R., Richter, B., Hassepass, F., Korvink, J.G., Echternach, M. (2015). A comparison of different methods to generate tooth surface models without applying ionizing radiation for digital 3-dimensional image fusion with magnetic resonance imaging-based data of the head and neck region. Journal of Computer Assisted Tomography, 39 (6), 882-889.

[21] Sicherer, S.H., Sampson, H.A. (2006). Food allergy. Journal of Allergy and Clinical Immunology, 117 (2), S470-S475.

[22] Weiger, M., Pruessmann, K.P., Bracher, A.K., Köhler, S., Lehmann, V., Wolfram, U., Hennel, F., Rasche, V. (2012). High-resolution ZTE imaging of human teeth. NMR in Biomedicine, 25 (10), 1144-1151.

[23] Ender, A., Mehl, A. (2013). Accuracy of complete-arch dental impressions: A new method of measuring trueness and precision. Journal of Prosthetic Dentistry, 109 (2), 121-128.

[24] Eggert, D.W., Lorusso, A., Fisher, R.B. (1997). Estimating 3-D rigid body transformations: A comparison of four major algorithms. Machine Vision and Applications, 9 (5-6), 272-290.

[25] Aalto, D., Aaltonen, O., Happonen, R.P., Jääsaari, P., Kivelä, A., Kuortti, J., Luukinen, J.M., Malinen, J., Murtola, T., Parkkola, R., Saunavaara, J., Soukka, T., Vainio, M. (2014). Large scale data acquisition of simultaneous MRI and speech. Applied Acoustics, 83, 64-75.

[26] Ojalammi, A., Malinen, J. (2017). Automated segmentation of upper airways from MRI - vocal tract geometry extraction. In Proceedings of the 10th International Joint Conference on Biomedical Engineering Systems and Technologies - Volume 2: Bioimaging. Setúbal, Portugal: SciTePress, 77-84.

[27] Athanasiou, A.E., Van der Meij, A.J.W. (1995). Posteroanterior (frontal) cephalometry. In Orthodontic Cephalometry. Mosby-Wolfe, 141-161.

[28] Lorensen, W.E., Cline, H.E. (1987). Marching cubes: A high resolution $3 \mathrm{D}$ surface construction algorithm. In SIGGRAPH '87: Proceedings of the 14th Annual Conference on Computer Graphics and Interactive Techniques. ACM, 163-169.

Received November 20, 2017. Accepted March 21, 2018. 